\title{
Metaheuristic Optimization Techniques for an Electromagnetic Multilayer Radome Design
}

\author{
Trung Kien Nguyen ${ }^{1} \cdot$ In-Gon Lee ${ }^{1} \cdot$ Obum Kwon $^{2} \cdot$ Yoon-Jae Kim $^{2} \cdot$ Ic-Pyo Hong ${ }^{1, *}$
}

\begin{abstract}
In this study, an effective method for designing an electromagnetic multilayer radome is introduced. This method is achieved by using ant colony optimization for a continuous domain in the transmission coefficient maximization with stability for a wide angle of incidence in both perpendicular and parallel polarizations in specific $\mathrm{X}$ - and $\mathrm{Ku}$-bands. To obtain the optimized parameter for a C-sandwich radome, particle swarm optimization algorithm is operated to give a clear comparison on the effectiveness of ant colony optimization for a continuous domain. The qualification of an optimized multilayer radome is also compared with an effective solid radome type in transmitted power stability and presented in this research.
\end{abstract}

Key Words: Ant Colony Optimization, Electromagnetic Multilayer Radome Design, Metaheuristic Optimization Algorithm, Particle Swarm Optimization, Transmission Coefficient Maximization.

\section{INTRODUCTION}

The problem of an electromagnetic multilayer dielectric design optimization for a frequency band and a desired range of incident angles has been introduced in recent years using such metaheuristic optimization algorithms as the genetic algorithm $[1,2]$, particle swarm optimization (PSO) [3, 4], or a hybrid algorithm that combines ant colony optimization (ACO) with the microgenetic algorithm [5]. In this study, an effective algorithm presented by Socha and Dorigo [6] in 2008 called ant colony optimization for a continuous domain $\left(\mathrm{ACO}_{\mathrm{R}}\right)$ is applied to design a $\mathrm{C}$-sandwich radome [7] with an applicable range of incident angle $\left(0^{\circ}-70^{\circ}\right)$ in both the transverse electric (TE) and transverse magnetic (TM) modes for the $\mathrm{X}$ - and $\mathrm{Ku}-$ bands. To validate the $\mathrm{ACO}_{\mathrm{R}}$ algorithm in a multilayer radome design optimization, the performance of the transmission coefficient characteristics of the $\mathrm{C}$-sandwich radome design optimized by $\mathrm{ACO}_{\mathrm{R}}$ has been compared with a conventional analysis method (i.e., a simpler and lower-cost analysis) that approximates multilayer radomes by a solid radome with an effective medium approximation (EMA) $[8,9]$. Then, the performance of $\mathrm{ACO}_{\mathrm{R}}$ is compared with a general optimization algorithm used in electromagnetic characteristic design (i.e., PSO). This study is organized as follows: Section II is an overview of the $\mathrm{ACO}_{\mathrm{R}}$ algorithm, which uses a boundary value method to evaluate the transmission coefficient of a multilayer radome. Section III examines the fitness function and compares the optimization results obtained by $\mathrm{ACO}_{\mathrm{R}}$ and $\mathrm{PSO}$. The simulation results and the transmitted power stability are compared with an effective solid radome, and the results are discussed. The conclusion is

Manuscript received July 26, 2018 ; Revised October 3, 2018 ; Accepted October 17, 2018. (ID No. 20180726-055J)

${ }^{1}$ Department of Information \& Communication Engineering, Kongju National University, Cheonan, Korea.

${ }^{2}$ Agency for Defense Development, Daejeon, Korea.

"Corresponding Author: Ic-Pyo Hong (e-mail: iphong@kongju.ac.kr)

This is an Open-Access article distributed under the terms of the Creative Commons Attribution Non-Commercial License (http://creativecommons.org/licenses/by-nc/4.0) which permits unrestricted non-commercial use, distribution, and reproduction in any medium, provided the original work is properly cited.

(c) Copyright The Korean Institute of Electromagnetic Engineering and Science. All Rights Reserved. 
provided in Section IV.

\section{METHODS}

\section{Ant Colony Optimization for a Continuous Domain}

Originally, ACO was introduced in 1992 by Dorigo [10], and it has been used to solve many combinational optimization problems consisting of a set of discrete decision variables. The idea of applying ACO in solving the continuous optimization problem was presented in 2008 by Socha and Dorigo [6]. The flowchart of the $\mathrm{ACO}_{\mathrm{R}}$ algorithm is illustrated in Fig. 1.

In $\mathrm{ACO}_{\mathrm{R}}$, the solutions are kept and sorted in a solution archive (Fig. 2), in which the associated weight of solution $l$ defined in Eq. (1) represents the strength of the solution in producing new solutions.

$$
\omega(l)=\frac{1}{q k \sqrt{2 \pi}} e^{-\frac{(l-1)^{2}}{2 q^{2} k^{2}}} .
$$

To generate new solutions, a kernel is selected by probability that is computed for each group as in Eq. (2).

$$
p(l)=\frac{\omega(l)}{\sum_{j=1}^{k} \omega(j)} .
$$

Roulette wheel selection [11] is applied to select a solution kernel. Each probability $p(l)$ is presented as a proportion of the wheel (Fig. 3), and a random selection process is made similarly to rotate the roulette wheel. An $m$ new random number

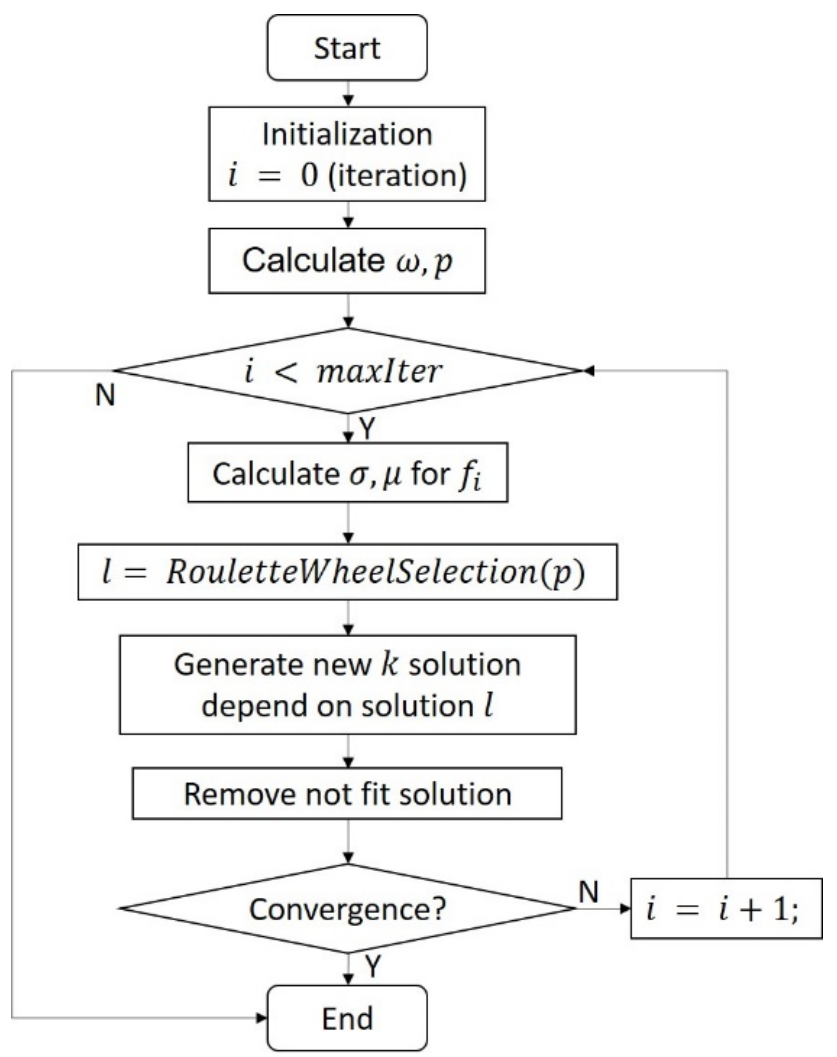

Fig. 1. Flowchart of $\mathrm{ACO}_{\mathrm{R}}$ algorithm.

\begin{tabular}{|c|c|c|c|c|c|}
\hline$s_{1}^{1}$ & $s_{1}^{2}$ & $\cdots$ & $s_{1}^{i}$ & $\cdots$ & $s_{1}^{n}$ \\
\hline$s_{2}^{1}$ & $s_{2}^{2}$ & $\cdots$ & $s_{2}^{i}$ & $\cdots$ & $s_{2}^{n}$ \\
\hline$\vdots$ & $\vdots$ & $\ddots$ & $\vdots$ & $\ddots$ & $\vdots$ \\
\hline$s_{l}^{1}$ & $s_{l}^{2}$ & $\cdots$ & $s_{l}^{i}$ & $\cdots$ & $s_{l}^{n}$ \\
\hline$\vdots$ & $\vdots$ & $\ddots$ & $\vdots$ & $\ddots$ & $\vdots$ \\
\hline$s_{k}^{1}$ & $s_{k}^{2}$ & $\cdots$ & $s_{k}^{i}$ & $\cdots$ & $s_{k}^{n}$ \\
\hline
\end{tabular}
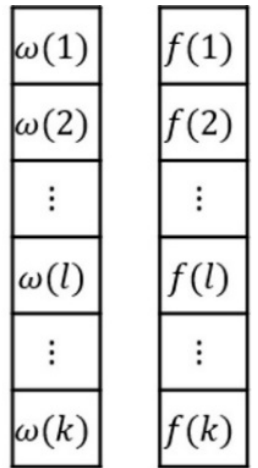

Fig. 2. Solution archive of the $A C O_{R}$ algorithm with $s_{l}^{i}$ as the value of parameter $i$ in group $l$ and the objective value $f(l)$ with associated weight $\omega(l)$.

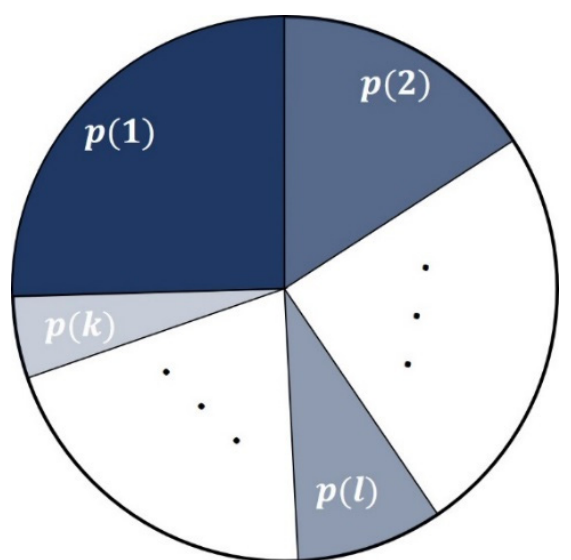

Fig. 3. Roulette wheel with a probability of solution $p(l)$ as the proportion of the wheel.

group according to the parameterized normal distribution is used with mean $\left(\mu_{l}^{i}\right)$ and standard deviation $\left(\sigma_{l}^{i}\right)$ for ant $i$ in group $l$.

$$
\begin{gathered}
\mu_{l}^{i}=s_{l}^{i} . \\
\sigma_{l}^{i}=\xi \sum_{j=1}^{k} \frac{\left|s_{j}^{i}-s_{l}^{i}\right|}{k-1} .
\end{gathered}
$$

$\xi>0$ is a constant parameter that is similar to the pheromone evaporation rate in ACO [10]. Then, $m$ new solutions are added to the solution archive and re-ordered. Non-fit solutions are removed, and only the $k$ best ones are kept after each iteration.

\section{Transmission Coefficient Characteristic of the C-Sandwich Radome}

A C-sandwich radome, which is a multilayer radome (style $\mathrm{d}$ ) [7], is considered in this work. The sandwich wall has five layers that were developed to cover an antenna array operating in a wide-band frequency range. The $\mathrm{C}$-sandwich construction consists of three skins that are interleaved by two cores. Typically, the relative permittivity of skin is greater than that of the core. To guarantee that the input and output of the propagation wave 
are equal, a $\mathrm{C}$-sandwich wall is designed with the first and last skins having the same thickness, and the widths of the two cores are the same as well. To fabricate a radome, the thickness of each layer is designed as the multiple of plies. The propagation wave on the radome with layer $i$ has thickness $d_{i}$, relative permittivity $\varepsilon_{i}$, loss tangent $\tan \delta_{i}$, and refractive index $n_{i}$. The $F_{j(j \in[1,7])} / B_{k(k \in[1,6])}$ are the forward and backward propagation waves, respectively. The forward and backward fields at the first left interface are related to Eq. (5).

$$
\begin{gathered}
{\left[\begin{array}{l}
F_{1} \\
B_{1}
\end{array}\right]=\prod_{i=1}^{5} \frac{1}{\tau_{i} \tau_{6}}\left[\begin{array}{cc}
e^{j k_{i} d_{i}} & r_{i} * e^{-j k_{i} d_{i}} \\
r_{i} * e^{j k_{i} d_{i}} & e^{-j k_{i} d_{i}}
\end{array}\right] \times} \\
{\left[\begin{array}{cc}
1 & r_{6} \\
r_{6} & 1
\end{array}\right]\left[\begin{array}{c}
F_{7} \\
0
\end{array}\right]}
\end{gathered}
$$

$\tau_{i}=1+r_{i}$ and $r_{i}$ is the intrinsic reflection coefficient of each interface and is calculated by the Fresnel equation. If one uses the formula in Eq. (5) and simplifies it to Eq. (6), the transmission coefficient can be obtained by Eq. (7).

$$
\begin{gathered}
{\left[\begin{array}{l}
F_{1} \\
B_{1}
\end{array}\right]=\left[\begin{array}{ll}
A_{11} & A_{12} \\
A_{21} & A_{22}
\end{array}\right]\left[\begin{array}{c}
F_{7} \\
0
\end{array}\right] .} \\
T=\frac{F_{7}}{F_{1}}=\frac{1}{A_{11}} .
\end{gathered}
$$

\section{RESULTS AND DISCUSSION}

\section{Fitness Function}

The main objective of this research is to maximize the transmission coefficient of a multilayer structure radome, which is calculated by Eq. (7) in a specific frequency band with various angles of incidence in both the TE and TM modes. To ensure the stability of the transmission coefficient, the following objective function is considered for optimization:

$$
\text { maximize } F=\{\min [T(f, \theta)]\}^{2} * 100 \text {, }
$$

where $T(f, \theta)$ is the transmission coefficient at frequency $f$ and incident angle $\theta$.

\section{Optimization Design Result}

The optimized thickness design is compared with an effective solid radome type with a half-wavelength thickness. The transmitted power is $80 \%$ greater in both polarizations, and the wide angle of incidence is the target of optimization. The design of the multilayer radome structure for optimization is shown in Table 1.

According to the EMA method, a multilayer radome can be approximated by its effective material property. The effective relative permittivity $\left(\varepsilon_{e f f}\right)$ of the radome after optimization is calculated by Eq. (9), where $n$ is the total number of layers, and $d_{i}, \varepsilon_{i}$ are the thickness and permittivity of layer $i$, respectively. By using a half-wavelength equation to assume the thickness of the effective medium, Eq. (10) can be used to compute
Table 1. C-sandwich radome layer properties

\begin{tabular}{lcccc}
\hline Layer & Permittivity & $\begin{array}{c}\text { Loss } \\
\text { tangent }\end{array}$ & $\begin{array}{c}\text { Thickness } \\
(\mathrm{mm})\end{array}$ & $\begin{array}{c}\text { Step/ply } \\
(\mathrm{mm})\end{array}$ \\
\hline Skin 1 & 4.4 & 0.016 & $0.48-2.4$ & 0.24 \\
Core 1 & 1.1 & 0.001 & $2-9$ & 0.1 \\
Skin 2 & 4.4 & 0.016 & $2.4-9.6$ & 0.24 \\
Core 2 & 1.1 & 0.001 & $2-9$ & 0.1 \\
Skin 3 & 4.4 & 0.016 & $0.48-2.4$ & 0.24 \\
\hline
\end{tabular}

the thickness of the effective half-wavelength solid-type radome at a resonant frequency of $10 \mathrm{GHz}$ for the X-band and $15 \mathrm{GHz}$ for the Ku-band with an angle of incidence $\theta=70^{\circ}$.

$$
\begin{gathered}
\varepsilon_{e f f} * \sum_{i=1}^{n} d_{i}=\sum_{i=1}^{n} \varepsilon_{i} * d_{i} . \\
d_{e f f}=\frac{\lambda}{2 * \sqrt{\varepsilon_{e f f}-\sin (\theta)^{2}}} .
\end{gathered}
$$

The first optimized radome of this study is the design for the $\mathrm{X}$-band (8-12 GHz) frequency. According to a comparative analysis with 30 execution times (Table 2), $\mathrm{ACO}_{\mathrm{R}}$ requires less time (56.69 seconds) to reach the convergence than PSO (70.18 seconds). Fig. 4 presents the comparison of transmitted power stability between the optimized multilayer radome and the effective solid radome with the stability of a multilayer radome in various angles, with both TE and TM modes being under 10\% change. The transmission coefficient characteristic of the Csandwich radome after optimization is presented in Fig. 5 in the X-band.

To give a more effective decision, the same design properties of the multilayer radome are applied in the Ku-band (12-18 $\mathrm{GHz})$. Table 3 shows the same result to demonstrate that $\mathrm{ACO}_{\mathrm{R}}$ has better performance than PSO. Fig. 6 illustrates that the stability in the transmitted power of the optimized multilayer structure is less than $10 \%$, which is outstanding compared with that of the effective solid radome. Fig. 7 shows the transmission coefficient of the optimized radome for the TE and TM modes in the Ku-band.

Table 2. Comparison of the 30 execution times between $\mathrm{ACO}_{\mathrm{R}}$ and PSO for the X-band frequency

\begin{tabular}{lcc}
\hline \multicolumn{1}{c}{ Detail } & $\mathrm{ACO}_{\mathrm{R}}$ & PSO \\
\hline Skin 1 $(\mathrm{mm})$ & 0.72 & 0.72 \\
Core 1 $(\mathrm{mm})$ & 5.0 & 5.0 \\
Skin 2 $(\mathrm{mm})$ & 7.68 & 7.68 \\
Core 2 $(\mathrm{mm})$ & 5.0 & 5.0 \\
Skin 3 $(\mathrm{mm})$ & 0.72 & 0.72 \\
Average time $(\mathrm{s})$ & 56.69 & 70.18 \\
Convergent iteration & 11 & 24 \\
\hline
\end{tabular}




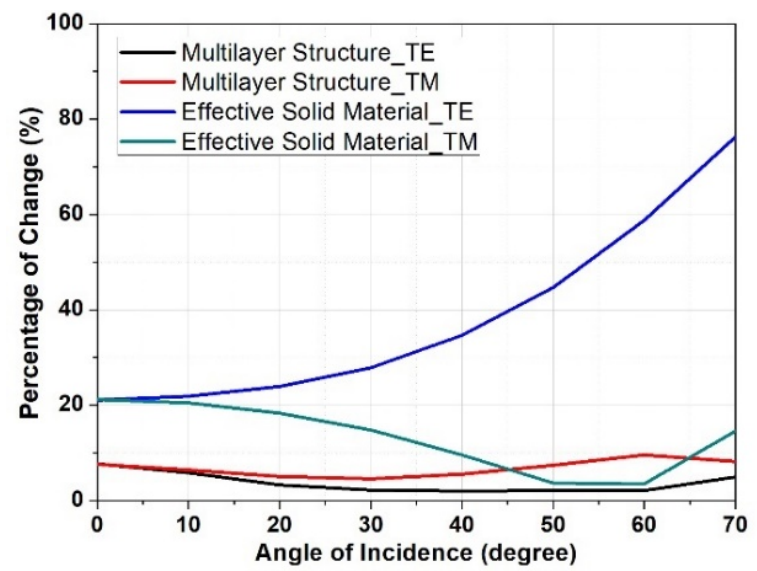

Fig. 4. Stability of the transmitted power of an optimized multilayer structure with an effective solid-type radome in the Xband.

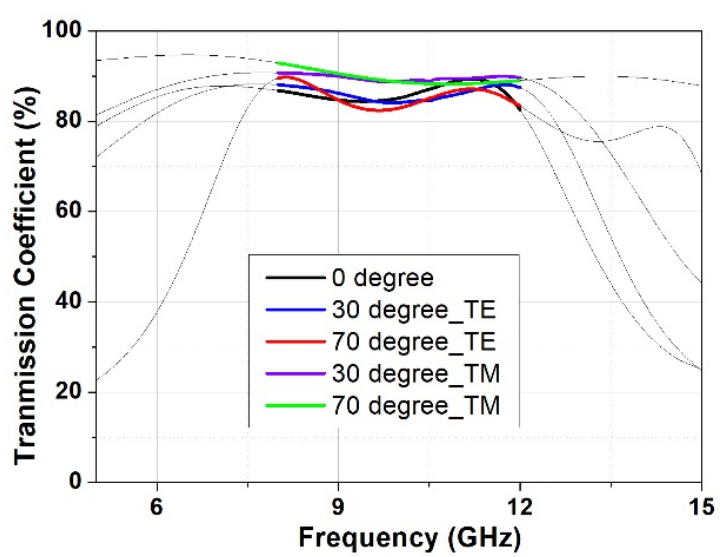

Fig. 5. Optimized transmission coefficient of the multilayer structure for the X-band.

According to the computed result, $\mathrm{ACO}_{\mathrm{R}}$ gives a promising value in optimizing multi-objective problems applied in the electromagnetic multilayer radome design.

\section{CONCLUSION}

A comparative study of two metaheuristic optimization algorithms, $\mathrm{PSO}$ and $\mathrm{ACO}_{\mathrm{R}}$, for an electromagnetic multilayer ra-

Table 3. Comparison of the 30 execution times between $\mathrm{ACO}_{\mathrm{R}}$ and $\mathrm{PSO}$ for the $\mathrm{Ku}$-band frequency

\begin{tabular}{lcc}
\hline \multicolumn{1}{c}{ Detail } & $\mathrm{ACO}_{\mathrm{R}}$ & $\mathrm{PSO}$ \\
\hline Skin 1 $(\mathrm{mm})$ & 0.48 & 0.48 \\
Core 1 $(\mathrm{mm})$ & 3.3 & 3.3 \\
Skin 2 $(\mathrm{mm})$ & 5.04 & 5.04 \\
Core 2 $(\mathrm{mm})$ & 3.3 & 3.3 \\
Skin 3 $(\mathrm{mm})$ & 0.48 & 0.48 \\
Average time $(\mathrm{s})$ & 64.13 & 71.86 \\
Convergent iteration & 12 & 24 \\
\hline
\end{tabular}

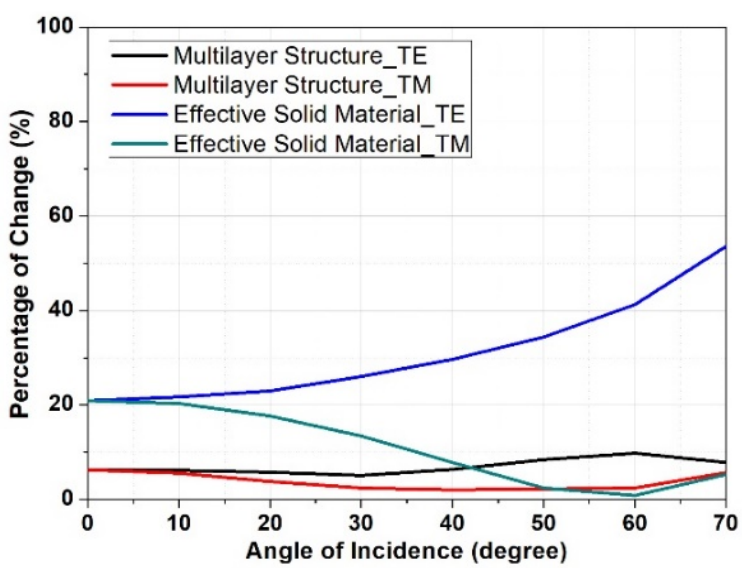

Fig. 6. Stability of the transmitted power of the optimized multilayer structure with an effective solid-type radome in the Ku-band.

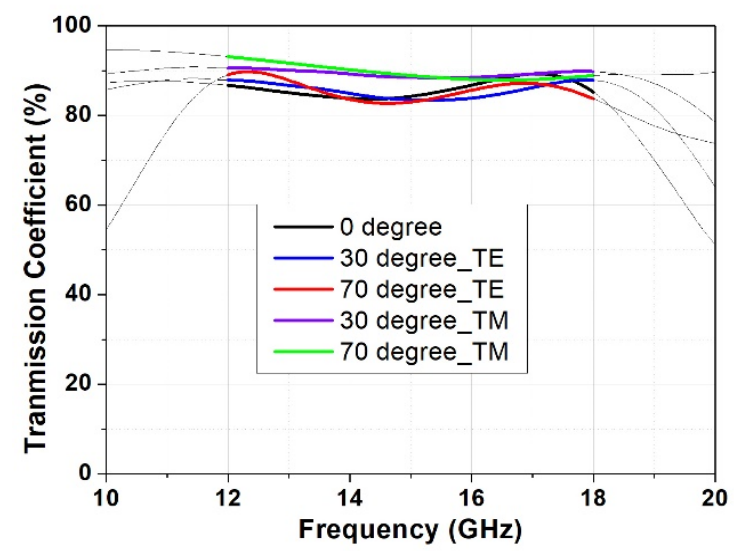

Fig. 7. Optimized transmission coefficient of the multilayer structure for the $\mathrm{Ku}$-band.

dome design with various angles of incidence $\left(0^{\circ}-70^{\circ}\right)$ in the perpendicular and parallel polarizations for the $\mathrm{X}$ - and $\mathrm{Ku}-$ bands was presented. The simulation results obtained by $A_{C O}$ were compared with those of a trusted optimization, PSO. The results strongly confirm that $\mathrm{ACO}_{\mathrm{R}}$ can be useful in the electromagnetic multilayer radome characteristic optimization. The stability for the transmission coefficient of the optimized structure is also guaranteed by $\mathrm{ACO}_{\mathrm{R}}$. In future works, the $\mathrm{ACO}_{\mathrm{R}}$ algorithm will be improved to solve more complex frequencyselective surface design optimization problems such as the $3 \mathrm{D}$ frequency-selective surface screen design.

This work was supported by the Agency for Defense Development under contract (No. UD170044JD).

\section{REFERENCES}

[1] H. A. El-Hakim, K. R. Mahmoud, and A. Abdelaziz, 
"Design of compact double-layer microwave absorber for $\mathrm{X}-\mathrm{Ku}$ bands using genetic algorithm," Progress In Electromagnetics Research, vol. 65, 157-168, 2016.

[2] N. Lassouaoui, H. Ouslimani, and A. Priou, "Genetic algorithms for automated design of the multilayer absorbers in the X-band and incident angle range," in Proceedings of 2008 Progress in Electromagnetics Research Symposium (PIERS), Hangzhou, China, 2008, pp. 509-513.

[3] K. W. Lee, I. P. Hong, B. J. Park, Y. C. Chung, and J. G. Yook, "Design of multilayer radome with particle swarm optimization," The Journal of Korean Institute of Electromagnetic Engineering and Science, vol. 21, no. 7, pp. 744-751, 2010.

[4] S. Roy, A. Mahanti, S. D. Roy, and G. K. Mahanti, "Comparison of evolutionary algorithms for optimal design of broadband multilayer microwave absorber for normal and oblique incidence," Applied Computational Electromagnetics Society Journal, vol. 31, no. 1, pp. 79-84, 2016.

[5] K. Chao, Y. Liu, and R. Yang, "Application of the hybrid algorithm combining ant colony optimization algorithm with microgenetic algorithm to the optimization of multi-

\section{Trung Kien Nguyen}

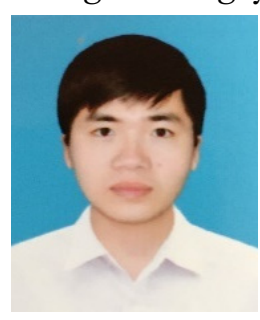

received his B.S. degree in information technology, University of Engineering and Technology from Vietnam National University, Hanoi, Vietnam, in 2013. He is currently working toward obtaining his M.S. degree in information and communication engineering from Kongju National University, Cheonan, Korea. His research interest is optimization algorithm in electromagnetics.

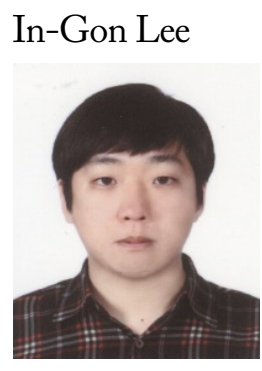

received his B.S. and M.S. degrees in information and communication engineering from Kongju $\mathrm{Na}^{-}$ tional University, Cheonan, Korea, in 2013 and 2015 , respectively. He is currently working toward obtaining his Ph.D. degree in information and communication engineering from the same university. His research interest is periodic electromagnetic structures.

layered radar absorbing coating," in Proceedings of International Conference on Microwave and Millimeter Wave Technology, Nanjing, China, 2008, pp. 597-600.

[6] K. Socha and M. Dorigo, "Ant colony optimization for continuous domains," European Journal of Operational Research, vol. 185, no. 3, pp. 1155-1173, 2008.

[7] D. J. Kozakoff, Analysis of Radome Enclosed Antennas. Boston, MA: Artech House, 1997.

[8] R. Lavin, "IEEE Phoenix Waves and Devices chapter," 2014; http://sites.ieee.org/phoenix-wad/files/2016/10/IEEE_ Lavin_Radomes_Final.pdf.

[9] X. Lei, L. Mao, Y. Lu, and P. Wang, "Revisiting the effective medium approximation in all-dielectric subwavelength multilayers: breakdown and rebuilding," Physical Review $B$, vol. 96, no. 3, article no. 035439, 2017.

[10] M. Dorigo, "Optimization, learning and natural algorithms," Ph.D. dissertation, Politecnico di Milano, Italy, 1992.

[11] D. E. Goldberg, Genetic Algorithms in Search, Optimization, and Machine Learning. Reading, MA: Addison-Wesley Publishing, 1989.

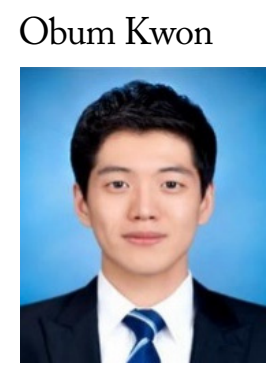

received his B.S. and M.S. degrees in mechanical engineering from Ulsan National Institute of Science and Technology, Ulsan, Korea, in 2015 and 2017, respectively. Since 2017, he has been a researcher for the Agency for Defense Development, Daejeon, Korea. His research interests include composite material structures and periodic electromagnetic structures.

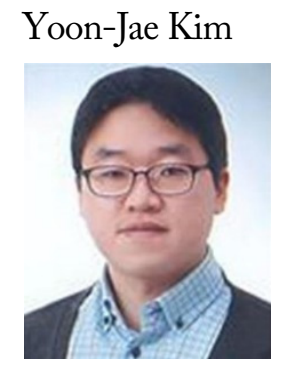

His research interests include aircraft structures and acoustic, vibration, and periodic electromagnetic structures. 


\section{Ic-Pyo Hong}

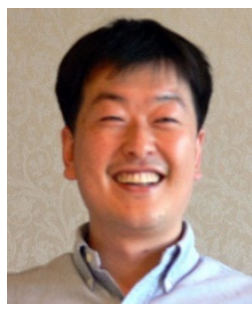

received his B.S., M.S., and Ph.D. degrees in electronics engineering from Yonsei University, Seoul, Korea, in 1994, 1996, and 2000, respectively. From 2000 to 2003, he was a senior engineer in CDMA Mobile Research at the Information and Communication Division of Samsung Electronics Company, Suwon, Korea. In 2006 and 2012, he was a visiting scholar at Texas A\&M University, College Station,

TX, USA, and Syracuse University, Syracuse, NY, USA, respectively. Since 2003, he has worked as a professor at the Department of Information and Communication Engineering, Kongju National University, Cheonan, Korea. His research interests include numerical techniques in electromagnetics, electromagnetic compatibility, and periodic electromagnetic structures. 